\title{
DIFERENTES AQUECIMENTOS NO DESEMPENHO DE REPETIÇÕES MÁXIMAS NA MUSCULAÇÃO
}

\author{
DIFFERENT WARM-UPS ON THE MAXIMUM REPETITION PERFORMANCE \\ IN RESISTANCE TRAINING \\ DIFERENTES CALENTAMIENTOS EN EL DESEMPEÑO DE REPETICIONES \\ MÁXIMAS EN LA MUSCULACIÓN
}

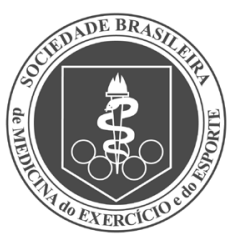

Artigo Original

\author{
Danilo Atanázio da Luz Junior ${ }^{3}$ \\ (Educador Físico) \\ Aylton Figueira Junor ${ }^{3}$ \\ (Educador Físico) \\ Érica Paes Serpa' (Educador Físico ) \\ Willy Andrade Gomes' \\ (Educador Físico) \\ Enrico Gori Soares ${ }^{1}$ (Educador Físico) \\ Charles Ricardo Lopes ${ }^{2}$ \\ (Educador Físico) \\ Luis Felipe Milano Teixeira ${ }^{4}$ \\ (Educador Físico ) \\ Paulo Henrique Marchetti $1^{1,2}$ \\ (Educador Físico) \\ 1. Departamento de Ciências do \\ Movimento Humano, Universidade \\ Metodista de Piracicaba, Piracicaba, \\ SP, Brasil. \\ 2. Instituto de Ortopedia e \\ Traumatologia, Faculdade de \\ Medicina, Universidade de São \\ Paulo, São Paulo, Brasil. \\ 3. Universidade São Judas Tadeu, \\ São Paulo, SP, Brasil. \\ 4. Faculdade de Educação Física da \\ UNIFIEO, Osasco, SP, Brasil.
}

\section{Correspondência:}

Paulo Henrique Marchetti Programa de Pós Graduação em Ciências do Movimento Humano, Faculdade de Ciências da Saúde, Universidade Metodista de Piracicaba (UNIMEP). Rodovia do Açúcar Km 156, Bloco 7, Sala 39, Taquaral. 13400-911. Piracicaba, SP, Brasil. pmarchetti@unimep.br

\section{RESUMO}

Introdução: Apesar do aquecimento ser considerado essencial na prática dos esportes, pouco se conhece sobre seu efeito no treinamento de força. Objetivo: Avaliar o efeito de diferentes estratégias de aquecimento no desempenho neuromuscular em adultos jovens submetidos a testes de repetições máximas de membro superior e inferior. Métodos: Dois experimentos foram realizados separadamente. Para o primeiro experimento, 16 adultos jovens hígidos realizaram um teste de repetições máximas a 70\% de 1RM no supino reto (previamente determinado). Quatro condições de aquecimento foram testadas: Controle (CON) sem aquecimento prévio, esteira (EST) 5' a $60 \% \mathrm{VO}_{2 \text { max }}$, resistência de força (REF) 15 repetições com 40\% de 1RM e força máxima (FM) duas séries de duas repetições com 90\% de 1RM. Para o segundo experimento, 14 adultos jovens hígidos, realizaram um teste de repetições máximas a 70\% de 1RM no leg press unilateral (previamente determinado). Cinco condições de aquecimento foram testadas: controle (CON) sem aquecimento prévio, esteira (EST) $5^{\prime}$ a $60 \% \mathrm{VO}_{2 \text { max }}$ bicicleta (BIC) 5' a 60\% VO 2max $_{\text {mat }}$ resistência de força (REF) 15 repetições com 40\% de 1RM e força máxima (FM) duas séries de duas repetições com 90\% de 1RM. Para ambos os experimentos o número de repetições realizadas e a percepção subjetiva de esforço foram avaliadas. Resultados: Para o primeiro experimento o protocolo FM foi superior a todos os outros protocolos. Não foram observadas diferenças entre as outras condições. Para o segundo experimento, foi observado diferenças entre todos os protocolos, exceto CON e EST. Sendo FM>BIC>REF>EST=CON. Conclusão: Os resultados do presente estudo sugerem que um aquecimento de força máxima levou a uma maior produção de força para os membros superiores e inferiores. Adicionalmente, aquecimentos aeróbios e de resistência de força apresentaram melhora no desempenho de força para os membros inferiores em uma menor magnitude.

Palavras-chave: desempenho esportivo, treinamento de resistência, força.

\section{ABSTRACT}

Introduction: Although the warm-up is considered essential for sports, little is known about its effect on strength training. Objective: To evaluate the effect of different warm-up strategies in neuromuscular performance in young adults undergoing maximum repetitions of upper and lower limb exercises. Methods: Two experiments were conducted separately. For the first experiment, 16 healthy young adults performed a test of maximal repetitions at 70\% 1RM in the bench press (previously determined). Four warm-up conditions were tested: control (CON) without prior warm-up, 5 min on the treadmill at $60 \% \mathrm{VO}_{2 \max }$ (EST), strength endurance (REF) with 1 set $\times 15$ repetitions with 40\% $1 \mathrm{RM}$ and maximal strength (MS) with two sets of two reps with 90\% 1RM. For the second experiment, 14 healthy young adults performed a maximal number of repetitions at 70\% IRM in the unilateral leg press (previously determined). Five warm-up conditions were tested: control (CON) without prior warm-up, 5 min of cycling at $60 \%$ VO2max (BIC), 5 min on the treadmill to $60 \% \mathrm{VO}_{2 \max }$ (EST), strength endurance (REF) with 1 set vs. 15 repetitions with 40\% 1RM and maximal strength (MS) with two sets of two reps with 90\% 1RM. For both experiments the number of repetitions performed and perceived exertion were evaluated. Results: For the first experiment, the MS condition was superior when compared to other protocols. No differences between the other conditions were observed. For the second experiment differences between all protocols were observed, except CON and EST, being MS $>B I C>R E F>=C O N$. Conclusion: The results of this study suggest that a warming-up of maximum strength may produce higher force production for the upper and lower limbs. Additionally, aerobic and strength resistance warm-up showed an improvement in strength performance for the lower limbs in a smaller magnitude.

Keywords: athletic performance, resistance training, force.

\section{RESUMEN}

Introducción: A pesar de que el calentamiento sea considerado esencial en la práctica de los deportes, poco se conoce sobre su efecto en el entrenamiento de fuerza. Objetivo: Evaluar el efecto de diferentes estrategias de calentamiento en el desempeño neuromuscular en adultos jóvenes sometidos a tests de repeticiones máximas de miembro superior e inferior. Métodos: Dos experimentos fueron realizados separadamente. Para el primer experimento, 16 adultos jóvenes 
hígidos realizaron un test de repeticiones máximas a 70\% de 1RM en el supino recto (previamente determinado). Cuatro condiciones de calentamiento fueron probadas: Control (CON) sin calentamiento previo, cinta ergonométrica (EST) $5^{\prime}$ a 60\% VO $\mathrm{V}_{2 \text { max }}$,esistencia de fuerza (REF) 15 repeticiones con 40\% de 1 RM y fuerza máxima (FM) dos series de dos repeticiones con 90\% de 1RM. Para el segundo experimento, 14 adultos jóvenes hígidos realizaron un test de repeticiones máximas a 70\% de 1RM en el leg press unilateral (previamente determinado). Cinco condiciones de calentamiento fueron

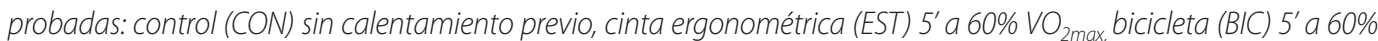
$V \mathrm{O}_{\text {max }}$ resistencia de fuerza (REF) 15 repeticiones con 40\% de 1RM y fuerza máxima (FM) dos series de dos repeticiones con 90\% de 1RM. Para ambos experimentos el número de repeticiones realizadas y la percepción subjetiva de esfuerzo fueron evaluadas. Resultados: Para el primer experimento el protocolo FM fue superior a todos los otros protocolos. No fueron observadas diferencias entre las otras condiciones. Para el segundo experimento, fueron observadas diferencias entre todos los protocolos, excepto CON y EST, siendo FM $>B I C>R E F>E S T=C O N$. Conclusión: Los resultados del presente estudio sugieren que un calentamiento de fuerza máxima llevó a una mayor producción de fuerza para los miembros superiores e inferiores. Adicionalmente, los calentamientos aeróbicos y de resistencia de fuerza presentaron mejora en el desempeño de fuerza para los miembros inferiores en una menor magnitud.

Palabras clave: desempeño deportivo, entrenamiento de resistencia, fuerza.

\section{INTRODUÇÃO}

O aquecimento é uma prática altamente difundida entre atletas, técnicos e praticantes de atividade física visando diminuir lesões ou preparar-se para a atividade subsequente ${ }^{1}$. Tradicionalmente, pode-se definir o aquecimento como um breve período onde exercícios aeróbios são seguidos por alongamentos e/ou exercícios específicos para determinada prática esportiva. Em relação ao desempenho, o aquecimento visa preparar o organismo para a melhor função durante a prática específica subsequente ${ }^{2,3}$. Diversos são os mecanismos responsáveis associados ao efeito do aquecimento na melhora do desempenho, como aumento da temperatura muscular, redução da rigidez muscular e articular, maior capacidade circulatória pulmonar, resultando na oferta de oxigênio nas vias aéreas e corrente sanguínea, aumento da sensibilidade da hemoglobina e mioglobina, elevação do consumo de oxigênio de base e aumento do fluxo sanguíneo muscular, ativação da atividade enzimática, facilitando a velocidade das reações metabólicas, aumento da taxa de condução nervosa, aumento da tensão de termorregulação, efeito de potencialização pós-ativação (PPA) e efeitos psicológicos ${ }^{1,4}$.

Apesar de o aquecimento ser considerado essencial na prática dos esportes, pouco se conhece sobre seu efeito no treinamento de força. Scharmann et al., ${ }^{5}$ investigaram os efeitos do aquecimento no teste de 1RM no leg press e supino e não verificaram aumento na produção de força, entretanto estudo de Barroso et al., ${ }^{6}$ observou que o aquecimento geral de longa duração e baixa intensidade resultou em melhora no desempenho durante o teste de 1RM. Em outro estudo, Serra et al., ${ }^{7}$ avaliou o efeito de alongamento estático na força máxima em diferente exercícios em indivíduos treinados e destreinados demonstrando que o alongamento promoveu efeito negativo de $17 \%$ na força máxima membros superiores e $8 \%$ em membros inferiores. Ainda demonstraram que indivíduos destreinados apresentaram os menores valores de força máxima em relação aos treinados. Na prática, diferentes estratégias de aquecimento específico são realizadas previamente ao treinamento de força, com a manipulação do número de repetições e intensidade (ex. alto número de repetições e baixa sobrecarga). Entretanto, não se conhece o real efeito destas estratégias no desempenho do treino.

O objetivo do presente estudo foi avaliar o efeito de diferentes estratégias de aquecimento no desempenho de repetições máximas na musculação em exercícios de membros superior e inferior.

\section{MATERIAIS E MÉTODOS}

Visando entender os efeitos do aquecimento no desempenho neuromuscular em membros superiores e inferiores, o presente estudo foi realizado como dois experimentos independentes. $O$ experimento 1 foi organizado com diferentes estratégias de aquecimento analisando o número máximo de repetições no exercício supino reto, e o experimento dois seguiu as mesmas estratégias, mas avaliando o desempenho no exercício leg press unilateral.

A amostra foi composta de adultos jovens, hígidos, do sexo masculino, aptos fisicamente para a realização do estudo e com experiência mínima em treinamento de força de 3 anos, utilizando os exercícios de supino reto e/ou leg press nos respectivos programas de treinamento. 0 experimento 1 utilizou o exercício supino reto; $n=16$, média de idade: $24 \pm 4$ anos; massa corporal: $75 \pm 7$ kg; estatura: $176 \pm 6$ cm; distância bi-acromial: $37 \pm 2$ cm; carga máxima no teste de 1RM: $89 \pm 17 \mathrm{~kg}$. 0 experimento 2 foi realizado com o exercício de leg press unilateral; $n=14$, média de idade: $27 \pm 6$ anos; massa corporal: $76 \pm 7$ kg; estatura: $176 \pm 8 \mathrm{~cm}$; carga máxima no teste de 1RM: $149 \pm 26$ kg. Os critérios de inclusão na composição amostral em ambos experimentos foram: (1) sem histórico prévio de cirurgia, lesão ou qualquer acometimento osteomioarticular em membros superiores e inferiores; (2) não terem treinado a área muscular envolvida nas 48 horas precedentes ao protocolo experimental; (3) não apresentar desordens neurológicas periféricas e/ou centrais.

Os voluntários assinaram o Termo de Consentimento Livre Esclarecido, respeitando as Diretrizes do CNS 196/96, sendo que todos os procedimentos foram aprovados pelo Comitê de Ética em Pesquisa da Universidade Metodista de Piracicaba, Piracicaba, SP, Brasil (Protocolo \#97/12).

\section{Procedimentos}

Experimento 1: Os sujeitos compareceram ao laboratório em quatro sessões distintas, com um intervalo de uma semana entre sessões. As coletas em cada uma das sessões foram realizadas sempre no mesmo dia da semana e horários, e pelo mesmo avaliador. Na primeira sessão foram coletados os dados antropométricos (estatura, massa, distância biacromial) e dados pessoais (nome, idade, tempo de prática na musculação). A distância biacromial foi utilizada para normatizar a distância entre as mãos de cada sujeito posicionadas (empunhadura) na barra do supino, sendo que todos realizaram a empunhadura com uma distância de 2 vezes a distância biacromial. Após a avaliação inicial, os sujeitos realizaram dois testes máximos de força: teste de 1RM no exercício supino reto ${ }^{8}$ e após 30 minutos de recuperação, os sujeitos realizaram um teste controle (CON), que consistiu na realização do número máximo de repetições sem aquecimento prévio contra uma sobrecarga externa de 70\%1RM (CON) até a falha mecânica concêntrica. Foram consideradas válidas apenas as repetições completas, caracterizadas pelo toque da barra na região torácica (fase excêntrica) e extensão completa dos cotovelos (fase concêntrica) ${ }^{9} \mathrm{~A}$ segunda, terceira 
e quarta sessões foram constituídas por três modelos distintos de aquecimento, aleatorizados, sendo: (1) esteira (EST): O indivíduo caminhou 5 min à $50 \% \mathrm{VO}_{2 \text { máx }}$ controlado por meio de um frequencímetro (Marca Oregon ${ }^{\circledR}$ ); (2) resistência de força (RF): 15 repetições à 40\%1RM e (3) força máxima (FM): duas séries de duas repetições com 90\%1RM. Após o aquecimento específico foi dado 1 min de intervalo de recuperação e um novo teste de repetições máximas com 70\%1RM foi realizado. Após o teste 70\%1RM, os sujeitos foram questionados sobre a percepção subjetiva de esforço (PSE) em uma escala que variou entre um e 10 (sendo, um muito fácil e 10 esforço máximo) $)^{10}$.

Experimento 2: Os sujeitos compareceram ao laboratório em cinco sessões distintas, com um intervalo de uma semana entre sessões. As coletas foram realizadas no mesmo dia e horários, pelo mesmo avaliador. Na primeira sessão foram coletados os dados antropométricos (estatura, massa) e dados pessoais (nome, idade, tempo de prática na musculação e dominância podal). Após a avaliação inicial, os sujeitos realizaram dois testes máximos de força: teste de 1RM no exercício leg press unilateral ${ }^{8}$ e após 30 minutos de recuperação os sujeitos realizaram o teste controle (CON) sendo o número máximo de repetições sem aquecimento contra uma sobrecarga externa de 70\%1RM (CON) até a falha mecânica concêntrica. Foi realizada uma marcação no equipamento garantindo que todos os sujeitos atingissem uma flexão máxima de joelhos de 90\%, de forma unilateral com o membro dominante. Foram consideradas válidas, apenas as repetições completas pelos critérios de alinhamento da marca adicionada ao trilho e a plataforma do equipamento levando a um ângulo de $90^{\circ}$ de flexão do joelho (fase excêntrica) e extensão completa do joelho (fase concêntrica) ${ }^{11}$. A segunda, terceira, quarta e quinta sessões foram constituídas por quatro modelos distintos de aquecimento, aleatorizados entre as sessões, sendo: (1) os indivíduos caminharam em uma esteira (EST) por 5 min à $50 \% \mathrm{VO}_{2 \text { máx }}$ controlado por meio de um frequencímetro (Marca Oregon ${ }^{\circledR}$ ); (2) os indivíduos pedalaram um cicloergômetro $(\mathrm{BIC})$ por 5 min à $50 \% \mathrm{VO}_{2 \text { máx }}$ controlado por meio de frequencímetro (Marca Oregon ${ }^{\circledR}$ ); (3) resistência de força (REF): 15 repetições com 40\%1RM e (4) força máxima (FM): duas séries de duas repetições com 90\%1RM. Após o aquecimento específico foi dado 1 min de intervalo e um novo teste com 70\% 1RM foi realizado, seguido por um questionamento oral sobre a PSE $^{10}$.

O número de repetições máximas atingidas no testeT70 e a PSE após cada condição de aquecimento foi quantificado e utilizado para verificar o efeito dos diferentes protocolos de aquecimento no desempenho neuromuscular em ambos os exercícios (supino reto e leg press unilateral).

\section{Análise estatística}

A normalidade e homogeneidade das variâncias foram verificadas utilizando o teste de Shapiro-Wilk e de Levene, respectivamente. Os dados analisados em valores médios e desvio padrão (DP). A ANOVA de medidas repetidas foi utilizada na comparação das diferenças entre as variáveis dependentes (Percepção subjetiva de esforço e o número máximo de repetições) nas diferentes condições experimentais para cada exercício. Um post hoc de Bonferroni (com correção) foi utilizado para verificar as diferenças entre estratégias. O cálculo do tamanho do efeito (software EffectSizeGenerator $2.3^{\circledR}$ ) foi realizado através da formula de Cohen (ES) e os resultados se basearam nos seguintes critérios: $<0,35$ sem expressão; 0,35-0,80 pequeno; 0,80-1,50 moderado; e > 1,5 grande, para sujeitos treinados de forma recreacional baseado em Rhea ${ }^{12}$.Uma significância de 5\% foi utilizada em todos os testes estatísticos.

\section{RESULTADOS}

O exercício supino reto apresentou diferenças significativas entre as seguintes estratégias de aquecimento: $\operatorname{CON} \times \operatorname{RF}(P=0,007), \operatorname{CON} x$ $\operatorname{FM}(P=0,014), \operatorname{FM} \times \operatorname{RF}(P=0,001), \operatorname{EST} \times \operatorname{RF}(P=0,001), \operatorname{EST} \times \mathrm{FM}(P=0,021)$ (figura 1). As comparações da magnitude de mudança são apresentadas na tabela 1. Não foram observadas diferenças significantes na PSE em nenhuma das condições experimentais (EST: $8 \pm 1$; RF: $8 \pm 1$; FM: $9 \pm 1$ ).

O exercício leg press unilateral demonstrou diferenças significativas entre as seguintes estratégias: $\operatorname{CON} \times \mathrm{FM}(P=0,001), \mathrm{BIC} \times \mathrm{FM}(P=0,008)$, $\operatorname{EST} \times \mathrm{FM}(P=0,001), \operatorname{RF} \times \mathrm{FM}(P=0,001)$ e BIC $\times \operatorname{EST}(P=0,005)$ (figura 2$)$. As comparações do tamanho do efeito são apresentadas na tabela 2. Não foram observadas diferenças significativas na PSE entre os protocolos de aquecimento (EST: $9 \pm 1 ; \mathrm{BIC}: 9 \pm 1 ; \mathrm{RF}: 9 \pm 1$; FM: $9 \pm 1$ ).

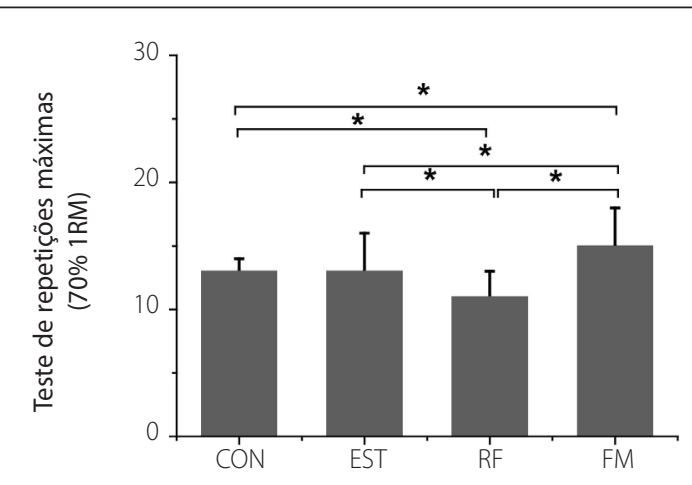

${ }^{*} \mathrm{P}<0,05 ;$ CON: controle; EST: esteira; RF: resistência de força; FM: força máxima.

Figura 1. Média e desvio padrão do número máximo de repetições para 70\%1RM após os protocolos de aquecimento para o exercício supino reto.

Tabela 1. Tamanho do efeito entre protocolos de aquecimento no exercício supino reto.

\begin{tabular}{c|c}
\hline Comparação entre condições & Tamanho do efeito \\
\hline CON $\times$ EST & 0,035 (trivial) \\
\hline CON $\times$ RF & 0,78 (pequeno) \\
\hline CON $\times$ FM & 0,78 (pequeno) \\
\hline EST $\times$ RF & 0,66 (pequeno) \\
\hline EST $\times$ FM & 0,66 (pequeno) \\
\hline RF $\times$ FM & 1,33 (moderado) \\
\hline
\end{tabular}

CON:controle; EST: esteira; RF: resistência de força; FM: força máxima.

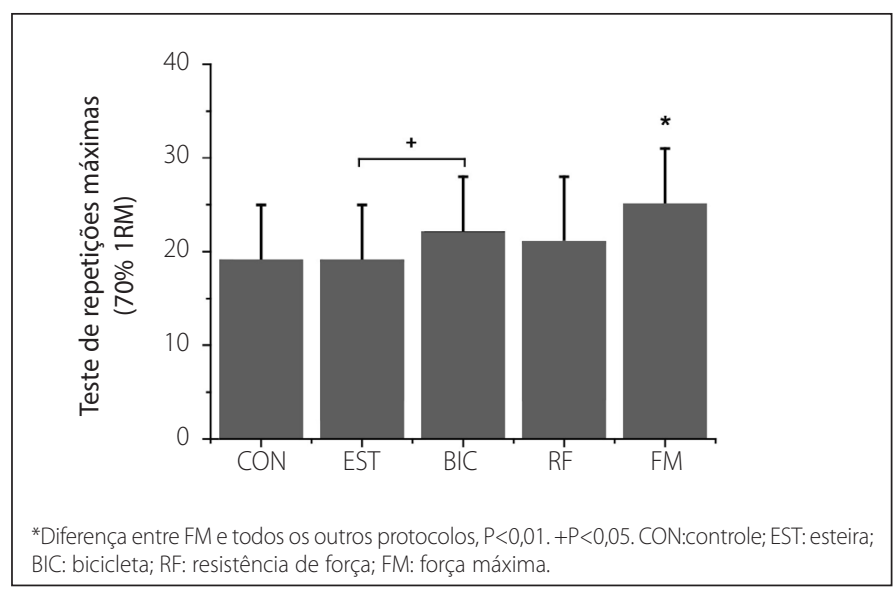

Figura 2. Média e desvio padrão do número máximo de repetições para 70\%1RM após os protocolos de aquecimento para o exercício leg press unilateral.

Tabela 2. Magnitude do efeito entre protocolos de aquecimento no exercício leg press unilateral.

\begin{tabular}{c|c}
\hline Comparação entre condições & Tamanho do Efeito \\
\hline CON $\times$ EST & 0,16 (trivial) \\
\hline CON $\times$ BIC & 0,50 (pequeno) \\
\hline CON $\times$ RF & 0,30 (trivial) \\
\hline CON $\times$ FM & 0,97 (moderado) \\
\hline EST $\times$ BIC & 0,46 (pequeno) \\
\hline EST $\times$ RF & 0,14 (trivial) \\
\hline EST $\times$ FM & 0,83 (moderado) \\
\hline BIC $\times$ RF & 0,15 (trivial) \\
\hline BIC $\times$ FM & 0,50 (pequeno) \\
\hline RF $\times$ FM & 0,61 (pequeno) \\
\hline CON: controle; EST: esteira; BIC: bicicleta; RF: resistência de força; FM: força máxima.
\end{tabular}




\section{DISCUSSÃO}

Diversos mecanismos são responsáveis pelo desempenho físico, sendo que as atividades específicas realizadas na rotina de aquecimento poderiam contribuir com o desempenho em um exercício, em função do aumento da temperatura corporal, aumento da viscosidade muscular, aumento ou melhora da taxa de condução nervosa, alteração para direita da curva de força-velocidade, possível aumento na provisão de energia anaeróbia, melhora na termorregulação, elevação do consumo inicial de oxigênio, acidose, potencialização pós-ativação (PPA), bem como efeitos psicológicos (estado de prontidão/atenção) 4,13.

Os resultados encontrados no experimento 1 demonstraram que 0 aquecimento realizado pelo protocolo de força máxima e resistência de força apresentaram maior rendimento quando comparados ao controle. Entretanto, o aquecimento de FM contribuiu com maior número de repetições (13\%) em relação ao RF e 27\% em relação à esteira. Considerando que em um programa de exercícios, não é comum utilizar a FM como uma estratégia de aquecimento, os dados encontrados sugerem que esta estratégia parece ser a mais eficaz em indivíduos experientes, embora muitos praticantes apontem que cargas menores sejam utilizadas como estratégia de aquecimento quando se objetiva a redução de lesões ${ }^{14}$. Hipotetizamos que a melhora no desempenho após o aquecimento em FM tenha ocorrido em função ao aumento da ativação dos precursores da capacidade de fosforilação das cadeias leves de miosina (aumentando sua sensibilidade ao $\mathrm{Ca}^{2+}$ ) e pela maior excitabilidade dos motoneurônios alfa $^{15-17}$. De acordo com Ament e Verkerke ${ }^{18}$ a capacidade de contração muscular é diretamente afetada por seu histórico contrátil, considerado como os efeitos residuais de atividades pregressas. A análise do número de repetições máximas no supino reto, não foi alterado quando precedido do aquecimento em esteira. Resultados similares foram encontrados por Raddi et al ${ }^{19}$ no qual não foram apresentadas diferenças na produção de força e número de repetições máximas no exercício supino reto, após 45 minutos em esteira rolante a $70 \%$ da $\mathrm{FC}_{\mathrm{Max}}$.

Os resultados do experimento 2, demonstraram um aumento significativo no número de repetições máximas comparando a estratégia de força máxima e as outras condições, fato similar ao encontrado no experimento 1. Adicionalmente, Weber, Brown et al. ${ }^{20}$ e McCann e Flanagan ${ }^{21}$ verificaram melhora do desempenho de saltos quando precedidos de exercícios de alta intensidade. Porém, no referido estudo não foram observadas diferenças entre a estratégia de resistência de força e a condição controle. O número total de repetições após o aquecimento de resistência de força foi $24 \%$ menor que o de FM, pelo fato da coexistência de mecanismos de melhora do desempenho,

\section{REFERÊNCIAS}

1. Fradkin AJ, Zazryn TR, Smoliga JM. Effects of warming-up on physical performance: a systematic review with metaanalysis. J Strength Cond Res. 2010;24(1):140-8.

2. Fradkin AJ, Zazryn TR, Smoliga JM. Effects of warming-up on physical performance: a systematic review with meta-analysis. J Strength Cond Res. 2010;24(1):140-8.

3. Bishop D. Warm up II: performance changes following active warm up and how to structure the warm up. Sports Med. 2003;33(7):483-98.

4. Bishop D. Warm up I: potential mechanisms and the effects of passive warm up on exercise performance. Sports Med. 2003;33(6):439-54.

5. Scharmann A, Eves S, Kimpel S. The effects of warm-up duration on 1-repetition maximum leg press/ bench press and push-ups to axhaustion. J Strength Cond Res. 2011;25(1):S79.

6. Barroso R, Silva-Batista C, Tricoli V, Roschel H, Ugrinowitsch C. The effects of different intensities and durations of the general warm-up on leg press 1RM. J Strength Cond Res. 2013;27(4):1009-13.

7. Serra AJ, Silva Jr JA, Marcolongo AA, Manchini MT, Oliveira JVA, Santos LFN, et al. Experience in resistance training does not prevent reduction in muscle strength evoked by passive static stretching. $J$ Strength Cond Res. 2013;27(8):2304-8.

8. Baechle TR, Earl RW. Fundamentos do Treinamento de Força e do Condicionamento Físico. São Paulo: Manole; 2010.

9. Marchetti PH, Arruda CC, Segamarchi LF, Soares EG, Ito DT, Da Luz Junior DA, et al. Exercício supino: uma breve revisão sobre os aspectos biomecânicos. Braz. J. Sports and Exerc. Res. 2010;1 (2):135-42.

10. Borg G, Ljunggren G, Ceci R. The increase of perceived exertion, aches and pain in the legs, heart rate and blood lactate during exercise on a bicycle ergometer. Eur J. Appl Physiol. 1985;54(4):343-9. associados à fadiga neuromuscular. Quanto aos protocolos de aquecimento aeróbio, os resultados no exercício leg press unilateral demonstram que somente o protocolo na bicicleta ergométrica apresentou efeito trivial na melhora do desempenho em relação ao CON. Barroso et al., ${ }^{22}$ analisaram o efeito do aquecimento em duas intensidades (40 e $70 \% V_{2 \max }$ ) e duração $\left(5^{\prime}\right.$ e $15^{\prime}$ ) no desempenho de força máxima (1RM) no exercício leg press. Dentre as combinações testadas, apenas a condição de intensidade moderada $\left(70 \% V \mathrm{O}_{2 \max }\right)$ e longa duração (15') promoveram queda no desempenho, sendo que na condição de baixa intensidade $\left(40 \% V_{2} O_{2 x}\right)$ e longa duração $\left(15^{\prime}\right)$ foi observada melhora na força máxima do exercício leg press. O presente estudo utilizou duração $\left(5^{\prime}\right)$ e intensidade $\left(50 \% \mathrm{VO}_{2 \text { max }}\right.$ ) similar ao estudo de Barroso et al. ${ }^{22}$, embora melhora no desempenho na força tenha sido encontrado apenas no protocolo de bicicleta quando comparado a esteira. Isto pode estar relacionado à maior amplitude de movimento e ações contráteis da musculatura envolvida na caminhada em esteira assim como equilíbrio nesta atividade. Por outro lado, sabe-se que intensidades mais altas promovem aumentos significativos na temperatura muscular, embora a maior depleção das reservas de fosfato de alta energia seja conhecida ${ }^{3}$, caso a intensidade esteja acima de $60 \%$ do $\mathrm{VO}_{2 \text { max }}$, fato que pode responder as diferenças encontradas entre protocolos.

Não foram observadas diferenças significativas em nenhum dos experimentos e das condições experimentais de aquecimento na PSE, sugerindo que os sujeitos não apresentaram diferentes percepções em relação às estratégias de aquecimento, possivelmente em função da relação volume e intensidade serem muito diferentes. Embora o presente estudo apresente limitações como a ausência de controle da temperatura muscular em relação às estratégias de aquecimento, avaliação das respostas mioelétricas e metabólicas, potencialmente influenciadas pelas diferentes estratégias de aquecimento, apresenta indicador aplicado em programas de exercícios, como o número de repetições, pois representa a relação colume-intensidade de treinamentos com pesos.

\section{CONCLUSÃO}

O aquecimento de força máxima para ambos os membros (superior e inferior) se apresentou efetivo na melhora do desempenho. Entretanto, a utilização da bicicleta apresentou melhora no desempenho de membros inferiores durante o leg press unilateral.

Todos os autores declararam não haver qualquer potencial conflito de interesses referente a este artigo.

11. Marchetti PH, Gomes WA, Da Luz Junior DA, Giampaoli B, Amorim MA, Bastos HL, et al. Aspectos neuromecânicos do exercício agachamento. Revista CPAQV. 2013;5(2):1-16.

12. Rhea MR. Determining the magnitude of treatment effects in strength training research through the use of the effect size. J Strength Cond Res. 2004;18(4):918-20.

13. Di Alencar TAM, Matias KFS. Princípios Fisiológicos do Aquecimento e Alongamento Muscular na Atividade Esortiva. Rev Bras Med Esporte. 2010;16(3):230-4.

14. Lin J, Chen T. Diversity of strength training methods: A theoretical approach. Strength Cond. J. 2012;34(2):42-9.

15. Hodgson M, Docherty D, Robbins D. Post-activation potentiation: underlying physiology and implications for motor performance. Sports Med. 2005;35(7):585-95. Epub 2005/07/20.

16. Ide BN, Lopes CR, Sarraipa MF. Fisiologia do treinamento esportivo: Força, potência, velocidade, resistência, periodização e habilidades psicológicas. São Paulo: Phorte Editora; 2010.

17. Lorenz D. Postactivation potentiation: an introduction. Int J Sports Phys Ther. 2011;6(3):234-40.

18. Ament W, Verkerke GJ. Exercise and fatigue. Sports Med. 2009;39(5):389-422.

19. Raddi LLO, Gomes RV, Charro MA, Bacurau RFP, Aoki MS. Treino de corrida não interfere no desempenho de força de membros superiores. Rev Bras Med Esporte. 2008;14(6):544-7.

20. Weber, KR; Brown,LE, et al. Acute Effects of Heavy-Load Squats on Consecutive Squat Jump Performance. J Strength Cond Res. 2008;22(3):726-30

21. McCann, MR; Flanagan,SP. The Effects of Exercise Selection and Rest Interval on Postactivation Potentiation of Vertical Jump Performance. J Strength Cond Res. 2010;24(5):1285-91.

22. Barroso R, Silva-Batista C, Tricoli V, Roschel H, Ugrinowitsch C. The effects of different intensities and durations of the general warm-up on leg press 1RM. J Strength Cond Res. 2013;27(4):1009-13. 